\title{
A Study on Nonlinear Control Strategy for Three-phase Voltage Source PWM DC/AC Inverter based on the PCH Model
}

\author{
Xiaobin Mu*, Jiuhe Wang*, and Xueyu Bao*
}

\begin{abstract}
The mathematical model of a three-phase voltage source pulse-width modulation (PWM) DC/AC inverter is non-linear, and in view of the traditional linear control strategy it can not meet the requirements of designing a high-performance inverter. What's more, when the loads are not pure resistive loads, the inverter further requires that the controller possess high-performance. This paper proposes a nonlinear control strategy for the inverter called Passivity-based Control. We can alter the inverter model in three-phase $a b c$ coordinate to two-phase synchronous rotating $d q$ coordinate for establishing the port-control Hamiltonian (PCH) model for this system. We can control the distribution of energy in the system to achieve the control aim. Simulation results show that the passivity-based control method can make this system possess a level of high-performance that is both robust and dynamic.
\end{abstract}

Keywords: Nonlinear control, PCH model, Passivity-based control

\section{Introduction}

With the mature development of power electronics te chnology, DC/AC inverters increasingly play an import ant role in the electrical field, in areas such as UPS, Wind and Photovoltaic Power Generation, Static Var C ompensators, Active Power Filters, etc. Application to these projects requires good dynamic performance and strong robustness of the inverter etc. In view of the mathematical model in which the inverter is non-linea $r$, a nonlinear control strategy from the nonlinear struct ure of the system could make the system achieve bette $\mathrm{r}$ performance. Passivity control theory is an important means for studying a nonlinear system. The overridin $\mathrm{g}$ idea is to make the controller redistribute reasonable energy of the system's output, and we can use this a pproach to improve system performance [1]. The passi vity control algorithm is calculated from the $\mathrm{PCH}$ mat hematical model based on passivity [2].

The paper is organized as follows. In Section II the DC/AC inverter model is set up. Section III attends to passivity based controller design. The simulation results are shown in Section IV. Finally, Section V states our conclusions.

\footnotetext{
* School of Automation, BIST University, China. (muxb2009@126.com, wjhyhrwm@163.com, baoxueyubxy@yahoo.cn)

Received 17 June 2011; Accepted 30 October 2011
}

\section{The DC/AC Inverter Model}

\subsection{The Topology of Three-phase Voltage PWM DC/AC} Inverter Systems

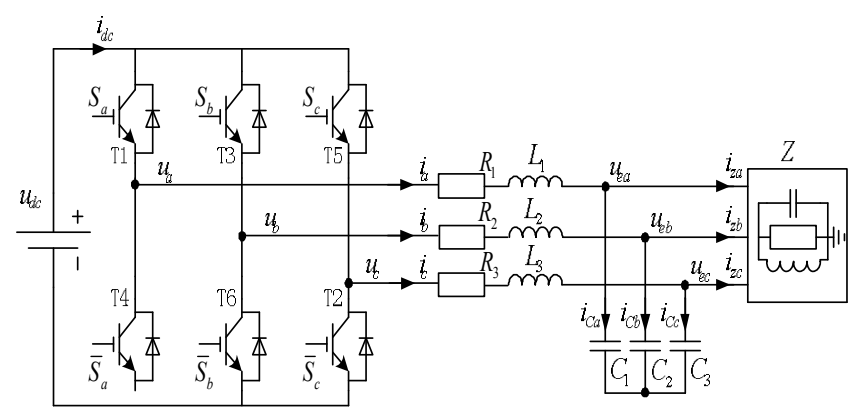

Fig. 1. The topology of the three-phase voltage PWM $\mathrm{DC} / \mathrm{AC}$ inverter system

The topology of Three-phase Voltage PWM DC/AC inverter systems is showed in the Figure 1. Assuming the resistance of the output reactor and the internal resistance of the system are seen as $R_{1}, R_{2}$ and $R_{3}, L_{1}, L_{2}$ and $L_{3}$ are the reactors. The impedances $Z$ is the three-phase load. $i_{a}, i_{b}$ and $i_{c}$ are the phase current of the inverter output. $i_{C a}$, $i_{C b}$ and $i_{C c}$ are the current that go into the capacitor. $i_{z a}$, $i_{z b}$ and $i_{z c}$ are the current that go into the loads, $u_{d c}$ is the DC voltage, $i_{d c}$ is the DC current provided by the DC voltage source. T1-T6 are for the IGBT switches. $S_{a}, S_{b}$ and $S_{c}$ are the drive signals for the up-bridge switches, $\bar{S}_{a}$, $\bar{S}_{b}$ and $\bar{S}_{c}$ are the drive signals for the down-bridge 
switches, $u_{a}, u_{b}$ and $u_{c}$ are the phase voltages of the inverter output, $u_{e a}, u_{e b}$ and $u_{e c}$ are the phase voltages of the filter output.

\subsection{The Mathematical Model of the DC/AC Inverter}

Assuming $\quad L_{1}=L_{2}=L_{3}=L \quad, \quad R_{1}=R_{2}=R_{3}=R \quad$, $C_{1}=C_{2}=C_{3}=C$. From Fig.1, we can obtain the mathematical model of the DC/AC inverter in three-phase $a b c$ coordinate that is

$$
\left\{\begin{array}{l}
L \frac{d i_{a}}{d t}=-R i_{a}+u_{d c}\left(S_{a}-\frac{1}{3} \sum_{j=a, b, c} S_{j}\right)-u_{e a}=-R i_{a}-u_{e a}+u_{a} \\
L \frac{d i_{b}}{d t}=-R i_{b}+u_{d c}\left(S_{b}-\frac{1}{3} \sum_{j=a, b, c} S_{j}\right)-u_{e b}=-R i_{b}-u_{e b}+u_{b} \\
L \frac{d i_{c}}{d t}=-R i_{c}+u_{d c}\left(S_{c}-\frac{1}{3} \sum_{j=a, b, c} S_{j}\right)-u_{e c}=-R i_{c}-u_{e c}+u_{c} \\
C \frac{d u_{e a}}{d t}=-i_{z a}+i_{a}, C \frac{d u_{e b}}{d t}=-i_{z b}+i_{b}, C \frac{d u_{e c}}{d t}=-i_{z c}+i_{c}
\end{array}\right.
$$

Where $S_{a}, S_{b}$ and $S_{c}$ are the switching functions of this inverter, When $S_{j}(j=a, b, c)=1$, the up-bridge is closed, the down-bridge is opened, $\bar{S}_{j}=1$ for the down-bridge is closed and the up-bridge is opend.

As existing time-varying signal of the voltage and the current in the three-phase coordinates to the mathematical model of the system, so we can transform it to a two-phase synchronous rotating coordinate for convenient calculation as follows

$$
\left\{\begin{array}{l}
L \frac{d i_{d}}{d t}=-R i_{d}+L \omega i_{q}+u_{d}-u_{e d}=-R i_{d}+L \omega i_{q}+S_{d} u_{d c}-u_{e d} \\
L \frac{d i_{q}}{d t}=-R i_{q}-L \omega i_{d}+u_{q}-u_{e q}=-R i_{q}-L \omega i_{d}+S_{q} u_{d c}-u_{e q} \\
C \frac{d u_{e d}}{d t}=-i_{z d}+C \omega u_{e q}+i_{d} \\
C \frac{d u_{e q}}{d t}=-i_{z q}-C \omega u_{e d}+i_{q}
\end{array}\right.
$$

Where, $S_{d}, S_{q}$ are the switching functions in the $d-q$ axis.

\subsection{The PCH Model of DC/AC Inverter}

Let $x_{1}=L i_{d}, x_{2}=L i_{q}, x_{3}=C u_{e d}, x_{4}=C u_{e q}$. (2) can be exp ressed as

$$
\left\{\begin{array}{l}
\frac{d x_{1}}{d t}=-R i_{d}+L \omega i_{q}+u_{d}-u_{e d}=-R \frac{x_{1}}{L}+\omega x_{2}+S_{d} u_{d c}-\frac{x_{3}}{C} \\
\frac{d x_{2}}{d t}=-R i_{q}-L \omega i_{d}+u_{q}-u_{e q}=-R \frac{x_{2}}{L}-\omega x_{1}+S_{q} u_{d c}-\frac{x_{4}}{C} \\
\frac{d x_{3}}{d t}=-i_{z d}+C \omega u_{e q}+i_{d}=-i_{z d}+\omega x_{4}+\frac{x_{1}}{L} \\
\frac{d x_{4}}{d t}=-i_{z q}-C \omega u_{e d}+i_{q}=-i_{z q}-\omega x_{3}+\frac{x_{2}}{L}
\end{array}\right.
$$

Let $H(x)=\frac{x_{1}^{2}}{2 L}+\frac{x_{2}^{2}}{2 L}+\frac{x_{3}^{2}}{2 C}+\frac{x_{4}^{2}}{2 C}$ denote the total energy of the DC/AC inverter [3]. Equation (3) can be expressed as

$$
\begin{gathered}
\dot{\boldsymbol{x}}=(\boldsymbol{J}-\mathfrak{R}) \frac{\partial H(\boldsymbol{x})}{\partial \boldsymbol{x}}+\boldsymbol{g}(\boldsymbol{x}) \boldsymbol{u} \\
\text { Where } \boldsymbol{J}=\left(\begin{array}{cccc}
0 & -L \omega & 1 & 0 \\
L \omega & 0 & 0 & 1 \\
-1 & 0 & 0 & -C \omega \\
0 & -1 & C \omega & 0
\end{array}\right), \boldsymbol{u}=\left(\begin{array}{c}
u_{d c} \\
u_{d c} \\
0 \\
0
\end{array}\right) \\
\Re=\left(\begin{array}{cccc}
R & 0 & 0 & 0 \\
0 & R & 0 & 0 \\
0 & 0 & i_{z d} / u_{e d} & 0 \\
0 & 0 & 0 & i_{z q} / u_{e q}
\end{array}\right), \boldsymbol{g}(\boldsymbol{x})=\left(\begin{array}{cccc}
S_{d} & 0 & 0 & 0 \\
0 & S_{q} & 0 & 0 \\
0 & 0 & 0 & 0 \\
0 & 0 & 0 & 0
\end{array}\right)
\end{gathered}
$$

(4) is the PCH model of the DC/AC inverter. With a skew symmetric matrix $\boldsymbol{J}$ expresses the interconnection structure of the system, i.e. $\boldsymbol{J}=-\boldsymbol{J}^{\boldsymbol{T}}$, and a symmetric positive semi-definite matrix $\mathscr{R}$ expresses the dissipation of the system.

\subsection{Design Procedure of the Controller}

We derive a PDE parameterized by the chosen matrices whose solutions characterize all the energy functions that can be assigned. Finally, from this family of solutions we choose one that satisfies the minimum requirement and compute the control. More precisely, the final objective is to find a static state-feedback control $\boldsymbol{u}=\boldsymbol{\beta}(\boldsymbol{x})$ such that the closed-loop dynamics is a PCH system with dissipation of the form [4]

$$
\dot{\boldsymbol{x}}=\left(\boldsymbol{J}_{d}(\boldsymbol{x})-\Re_{d}(\boldsymbol{x})\right) \frac{\partial H_{d}(\boldsymbol{x})}{\partial \boldsymbol{x}}
$$

Where the new energy function $H_{d}(\boldsymbol{x})$ has a strict local minimum at the desired equilibrium $\boldsymbol{x}^{*}, \boldsymbol{J}_{d}(\boldsymbol{x})=-\boldsymbol{J}_{d}^{\mathrm{T}}(\boldsymbol{x})$ 
and $\boldsymbol{R}_{d}(\boldsymbol{x})=\mathfrak{R}_{d}^{\mathrm{T}}(\boldsymbol{x}) \geq 0$ are some desired interconnection and damping matrices, respectively.

Given $\boldsymbol{J}, \mathfrak{R}, H, \boldsymbol{g}$ and the desired equilibrium to be stabilized $x^{*} \in \mathrm{R}^{\mathrm{n}}$, we can assume that we can find the functions $\boldsymbol{\beta}(\boldsymbol{x}), \boldsymbol{J}_{a}(\boldsymbol{x}), \Re_{a}(\boldsymbol{x})$ and a vector function $\boldsymbol{K}(\boldsymbol{x})$ satisfying

$$
\begin{aligned}
& {\left[\boldsymbol{J}(\boldsymbol{x}, \boldsymbol{\beta}(\boldsymbol{x}))+\boldsymbol{J}_{a}(\boldsymbol{x})-\left(\mathfrak{R}(\boldsymbol{x})+\mathfrak{R}_{a}(\boldsymbol{x})\right)\right] \boldsymbol{K}(\boldsymbol{x})} \\
& =-\left[\boldsymbol{J}_{a}(\boldsymbol{x})-\mathfrak{R}_{a}(\boldsymbol{x})\right] \frac{\partial H}{\partial \boldsymbol{x}}(\boldsymbol{x})+\boldsymbol{g}(\boldsymbol{x}, \boldsymbol{\beta}(\boldsymbol{x}))
\end{aligned}
$$

and such that [5]

(i) (Structure preservation)

$$
\begin{aligned}
& \boldsymbol{J}_{d}(\boldsymbol{x})=\boldsymbol{J}(\boldsymbol{x}, \boldsymbol{\beta}(\boldsymbol{x}))+\boldsymbol{J}_{a}(\boldsymbol{x})=-\left[\boldsymbol{J}(\boldsymbol{x}, \boldsymbol{\beta}(\boldsymbol{x}))+\boldsymbol{J}_{a}(\boldsymbol{x})\right]^{\mathrm{T}} \\
& \boldsymbol{R}_{d}(\boldsymbol{x})=\mathfrak{R}(\boldsymbol{x})+\mathfrak{R}_{a}(\boldsymbol{x})=\left[\Re(\boldsymbol{x})+\Re_{a}(\boldsymbol{x})\right]^{\mathrm{T}} \geq 0
\end{aligned}
$$

(ii) (Integrability) $\boldsymbol{K}(\boldsymbol{x})$ is the gradient of a scalar function. That is

$$
\frac{\partial \boldsymbol{K}(\boldsymbol{x})}{\partial \boldsymbol{x}}=\left(\frac{\partial \boldsymbol{K}(\boldsymbol{x})}{\partial \boldsymbol{x}}\right)^{\mathrm{T}}
$$

(iii) (Equilibrium assignment) $\boldsymbol{K}(\boldsymbol{x})$, at $\mathrm{x}^{*}$, verifies

$$
\boldsymbol{K}\left(\boldsymbol{x}^{*}\right)=-\frac{H\left(\boldsymbol{x}^{*}\right)}{\partial \boldsymbol{x}}
$$

(iv) (Lyapunov stability) The Jacobian of $\boldsymbol{K}(\boldsymbol{x})$, at $\mathrm{x}^{*}$, satisfies the bound

$$
\frac{\partial \boldsymbol{K}\left(\boldsymbol{x}^{*}\right)}{\partial \boldsymbol{x}}>-\frac{\partial^{2} H\left(\boldsymbol{x}^{*}\right)}{\partial \boldsymbol{x}^{2}}
$$

Under these conditions, the closed-loop system $\boldsymbol{u}=\boldsymbol{\beta}(\boldsymbol{x})$, will be a PCH system with dissipation of the form(5), where $H_{d}(\boldsymbol{x})=H(\boldsymbol{x})+H_{a}(\boldsymbol{x}), \partial H_{a}(\boldsymbol{x}) / \partial \boldsymbol{x}=\boldsymbol{K}(\boldsymbol{x})$

Furthermore, $\mathrm{x}^{*}$ will be a locally stable equilibrium of th e closed-loop.

\section{Controller Design}

According to (4) $S_{d}$ and $S_{q}$ are achieved to realize $\boldsymbol{x}^{*}=\arg \min H_{d}(\boldsymbol{x})$ under conditions (i), (ii), (iii) and (iv). The matching objective is achieved if and only if
$\left(\boldsymbol{J}_{d}(\boldsymbol{x})-\boldsymbol{R}_{d}(\boldsymbol{x})\right) \frac{\partial H_{a}(\boldsymbol{x})}{\partial \boldsymbol{x}}+\left(\boldsymbol{J}_{a}(\boldsymbol{x})-\boldsymbol{R}_{a}(\boldsymbol{x})\right) \frac{\partial H(\boldsymbol{x})}{\partial \boldsymbol{x}}-\boldsymbol{g u}=\mathbf{0}$

where $\boldsymbol{J}_{d}(\boldsymbol{x})=\boldsymbol{J}(\boldsymbol{x})+\boldsymbol{J}_{a}(\boldsymbol{x}), \mathfrak{R}_{d}(\boldsymbol{x})=\mathfrak{R}(\boldsymbol{x})+\mathfrak{R}_{a}(\boldsymbol{x})$, $H_{d}=H(\boldsymbol{x})+H_{a}(\boldsymbol{x})$. Defining

$$
\begin{aligned}
\boldsymbol{K}(\boldsymbol{x}) & =\left[\begin{array}{llll}
k_{1}(x) & k_{2}(x) & k_{3}(x) & k_{4}(x)
\end{array}\right]^{\mathrm{T}} \\
& =\left(\begin{array}{llll}
\partial H_{a} / \partial x_{1} & \partial H_{a} / \partial x_{2} & \partial H_{a} / \partial x_{3} & \partial H_{a} / \partial x_{4}
\end{array}\right)^{\mathrm{T}}
\end{aligned}
$$

and fixing the interconnection and damping matrices $\boldsymbol{J}_{d}(\boldsymbol{x})=\boldsymbol{J}(\boldsymbol{x})$ and $\boldsymbol{R}_{d}(\boldsymbol{x})=\boldsymbol{R}(\boldsymbol{x})$, that is $\boldsymbol{J}_{a}(\boldsymbol{x})=\mathbf{0}$ and $\Re_{a}(\boldsymbol{x})=\mathbf{0}$. Equation (7) simplifies to

$$
(\boldsymbol{J}(\boldsymbol{x})-\Re(\boldsymbol{x})) \partial H_{a}(\boldsymbol{x}) / \partial \boldsymbol{x}-\boldsymbol{g u}=\mathbf{0}
$$

From (9), we obtain the equation expressing $S_{d}$ and $S_{q}$ as follows

$$
\left\{\begin{array}{l}
S_{d}=\left(k_{2} L \omega-k_{3}-k_{1} R\right) / u_{d c} \\
S_{q}=\left(-k_{1} L \omega-k_{4}-k_{2} R\right) / u_{d c}
\end{array}\right.
$$

In order to simply the design of the controller, we can take $k_{1}=k_{1}\left(x_{3}\right), k_{2}=k_{2}\left(x_{3}\right), k_{3}=k_{3}\left(x_{3}\right), k_{4}=k_{4}\left(x_{3}\right), \quad$ and from ii, we get $k_{3}=A_{3}, k_{4}=A_{4}$ under the integrability condition (ii), and $A_{3}$ and $A_{4}$ are constants [6].

In order to obtain the extremum of $H_{d}(\boldsymbol{x})$ at $\boldsymbol{x}^{*}=\left(\begin{array}{llll}x_{1}^{*} & x_{2}^{*} & x_{2}^{*} & x_{4}^{*}\end{array}\right)^{\mathrm{T}}$, the equilibrium condition is

$$
\frac{\partial H_{d}(\boldsymbol{x})}{\partial \boldsymbol{x}}=\mathbf{0} \Rightarrow \begin{cases}x_{1}^{*} / L+k_{1}=0 & x_{2}^{*} / L+k_{2}=0 \\ x_{3}^{*} / C+A_{3}=0 & x_{4}^{*} / C+A_{4}=0\end{cases}
$$

With $x_{1}^{*}=L I_{\text {ref }}, x_{2}^{*}=0, x_{3}^{*}=C U_{\text {ref }}, x_{4}^{*}=0$, from (9) and (11), $k_{1}, k_{2}, k_{3}$ and $k_{4}$ are expressed as

$$
\left.\begin{array}{l}
\left\{\begin{array}{l}
k_{3}=A_{3}=-x_{3}^{*} / C=-U_{r e f} \\
k_{4}=A_{4}=-x_{4}^{*} / C=0
\end{array}\right. \\
\left\{\begin{array}{l}
k_{1}+k_{4} C \omega-k_{3} i_{z d} / u_{e d}=0 \\
k_{2}-k_{3} C \omega-k_{4} i_{z q} / u_{e q}=0
\end{array}\right\}
\end{array}\right\}\left\{\begin{array}{l}
k_{1}=-\frac{U_{r e f} i_{z d}}{u_{e d}} \\
k_{2}=-U_{r e f} C \omega
\end{array}\right.
$$

(12) satisfies condition (iii) based on power conservation $U_{\text {ref }}=I_{\text {ref }} R_{L}$ at $\boldsymbol{x}^{*}$. From (12) and (8) $H_{a}(\boldsymbol{x})$ is

$$
H_{a}(\boldsymbol{x})=-\left(U_{r e f} i_{z d} / u_{e d}\right) x_{1}-U_{r e f} C \omega x_{2}-U_{r e f} x_{3}
$$


In order to guarantee that $H_{d}(\boldsymbol{x})$ has a minimum at $\boldsymbol{x}$, th e Hessian of $H_{d}(\boldsymbol{x})$ has to obey $\left.\frac{\partial^{2} H_{d}}{\partial \boldsymbol{x}^{2}}\right|_{\boldsymbol{x}=\boldsymbol{x}^{*}}>0$. From (13) a nd (7) we can obtain that

$$
\left.\frac{\partial^{2} H_{d}}{\partial \boldsymbol{x}^{2}}\right|_{x=x^{*}}=\left(\begin{array}{cccc}
1 / L & 0 & 0 & 0 \\
0 & 1 / L & 0 & 0 \\
0 & 0 & 1 / C & 0 \\
0 & 0 & 0 & 1 / C
\end{array}\right)
$$

It is always a positive definite matrix, so the minimum condition is satisfied. Substituting (12) into (10), we can obtain the control laws

$\boldsymbol{\beta}(\boldsymbol{x})=\left(\begin{array}{l}S_{d} \\ S_{q}\end{array}\right)=\left(\begin{array}{l}\left(-U_{r e f} C \omega^{2} L+U_{r e f}+U_{r e f} i_{z d} R / u_{e d}\right) / u_{d c} \\ \left(U_{r e f} i_{z d} L \omega / u_{e d}+U_{r e f} C \omega R\right) / u_{d c}\end{array}\right)$

So we can obtain the inverter structure diagram based on a microcomputer with passivity control as Figure 2.

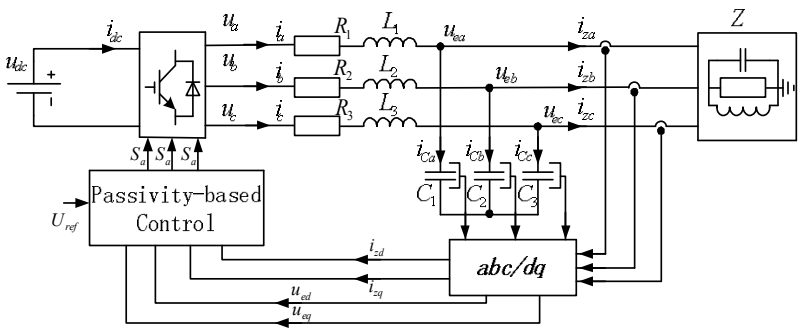

Fig. 2. Inverter structure diagram based on microcomputer

\section{Simulation of System}

We simulate the system by using SIMULINK tools in MATALAB and assume the DC voltage as $600 \mathrm{~V}$, the resistance of the output reactor and the internal resistance of the system $\mathrm{R}$ as $0.2 \Omega$, output reactor $\mathrm{L}$ as $10 \mathrm{mH}$, and the capacitor as $2200 \mu \mathrm{F}$.

We set the desired voltage in the d-axis and q-axis of three-phase output phase voltage with $311 \mathrm{~V}$ and $0 \mathrm{~V}$, and when the load alters from $20 \Omega$ to $10 \Omega$ at 0.02 seconds, from $10 \Omega$ to $20 \Omega$ at 0.04 seconds, the waveforms are shown in Figure 3. The voltage waveforms of three-phase output phase voltage, which under the conditions of Figure 3, are shown in Figure 4. From Figure 3 and Figure 4, we can see that the system transition time is very short and the tracking effect ideal. When the load alters from $20 \Omega$ to $10 \Omega / 2 \mathrm{H}$ at 0.02 seconds, from $10 \Omega / 2 \mathrm{H}$ to $20 \Omega$ at 0.04 seconds, the voltage waveforms in the d-axis and q-axis are shown in Figure 5, the voltage waveforms of three-phase output phase voltage are shown in Figure 6. When the DC voltage is disturbed by random noise and the load alters from $20 \Omega$ to $10 \Omega / 2 \mathrm{H}$ at 0.02 seconds and from $10 \Omega / 2 \mathrm{H}$ to $20 \Omega$ at 0.04 seconds, the voltage waveforms in the $\mathrm{d}$-axis and q-axis are shown in Figure 7. The voltage waveforms of three-phase output phase voltage, which under the conditions of Figure 7, are shown in Figure 8. From Figure 7 and Figure 8, we can see that the system possesses a level of highperformance that is both robustness and dynamic.

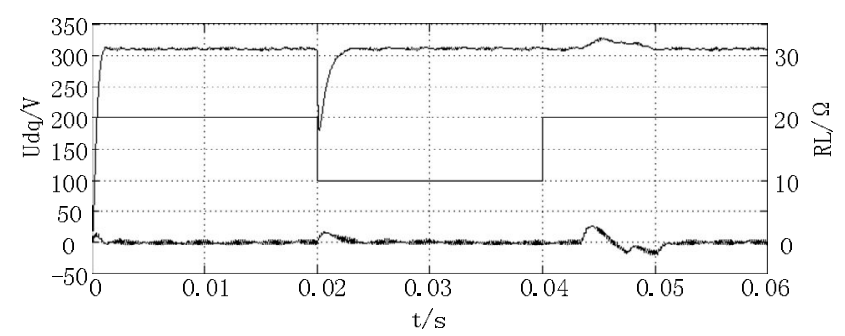

Fig. 3. The voltage waveforms in the d-axis and q-axis of three-phase output phase voltage When the load alters from $20 \Omega$ to $10 \Omega$ at 0.02 seconds, and from $10 \Omega$ to $20 \Omega$ at 0.04 seconds.

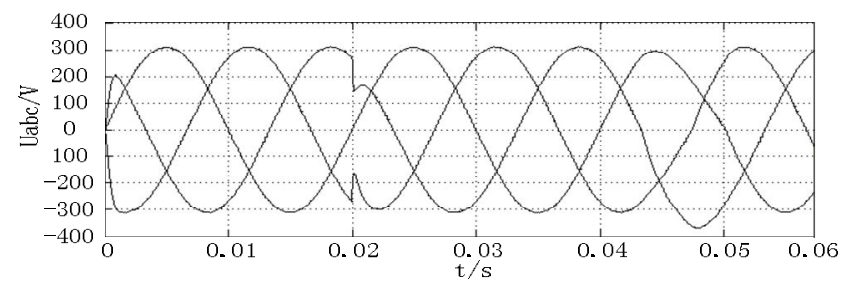

Fig. 4. The voltage waveforms of three-phase output phase voltage when the load alters from $20 \Omega$ to $10 \Omega$ at 0.02 seconds, and from $10 \Omega$ to $20 \Omega$ at 0.04 seconds.

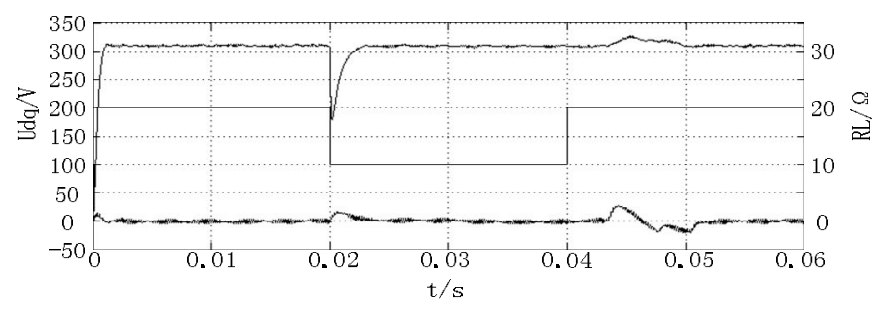

Fig. 5. The voltage waveforms in the d-axis and q-axis of three-phase output phase voltage when the load alters from $20 \Omega$ to $10 \Omega / 2 \mathrm{H}$ at 0.02 seconds, and from $10 \Omega / 2 \mathrm{H}$ to $20 \Omega$ at 0.04 seconds.

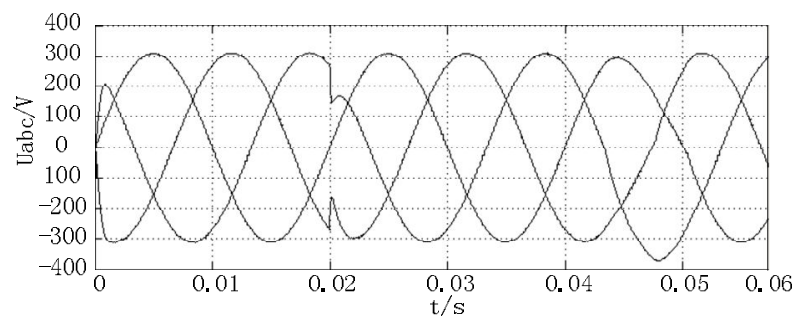

Fig. 6. The voltage waveforms of three-phase output phase voltage When the load alters from $20 \Omega$ to $10 \Omega / 2 \mathrm{H}$ at 0.02 seconds, and from $10 \Omega / 2 \mathrm{H}$ to $20 \Omega$ at 0.04 seconds. 


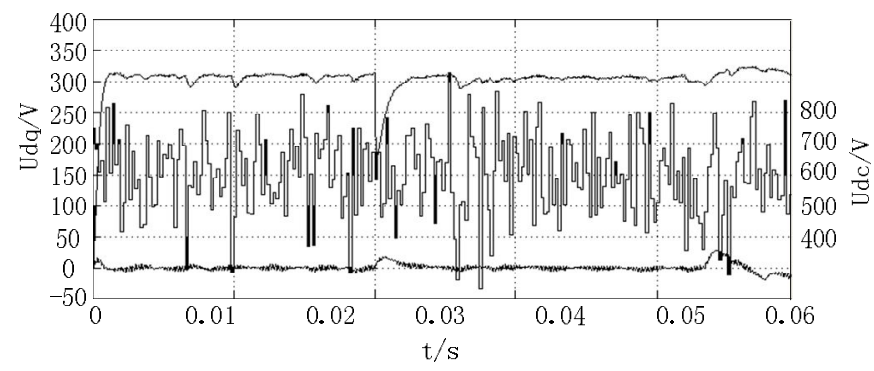

Fig. 7. The voltage waveforms with the DC voltage is disturbed by random noise and the load alters from $20 \Omega$ to $10 \Omega / 2 \mathrm{H}$ at 0.02 seconds and from $10 \Omega / 2 \mathrm{H}$ to $20 \Omega$ at 0.04 seconds.

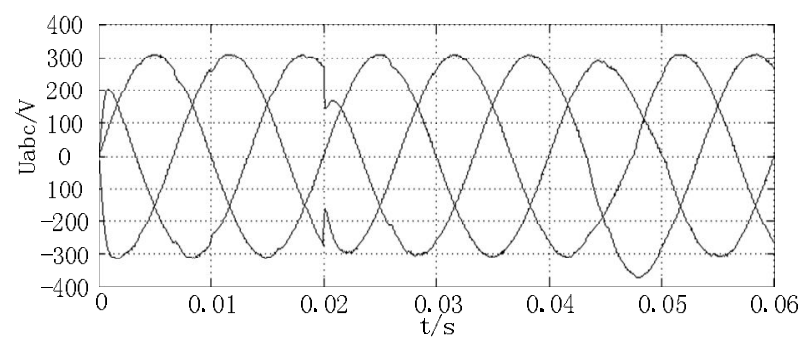

Fig. 8. The voltage waveforms with the DC voltage is disturbed by random noise and the load alters from $20 \Omega$ to $10 \Omega / 2 \mathrm{H}$ at 0.02 seconds and from $10 \Omega / 2 \mathrm{H}$ to $20 \Omega$ at 0.04 seconds.

\section{Conclusions}

Designing a passivity controller is simple, it has few adjustable parameters, and it's control effect is better. From the simulation effect, the passivity controller can make the system possess the high-performance that is both robustness and dynamic. This study on passive control theory applied in the $\mathrm{DC} / \mathrm{AC}$ inverter system has important guiding significance for the specific design.

\section{Acknowledgements}

This work is supported by National Natural Science Foundation of China (51077005)/funding project for academic human resources development in institutions of higher learning under the jurisdiction of Beijing municipality (PHR201007130).

\section{References}

[1] Xiaobin $\mathrm{Mu}$, Jiuhe Wang, Wen Gu, Shengsheng Xu. Passivity-Based of Photovoltaic Grid-Connected Inverter Based on Nonlinear Current Control Strategy. Symposium on Power Electronics \& Electrical Drives Proceedings, pp. 298302,April 2011.

[2] Alejandro Donaire, Sergio Junco. "Energy Shaping Interconnection and Damping Assignment and Integral
Control in the Bond Graph Domain". Simulation Modeling Practice and Theory, 2009, pp. 152-174

[3] Dazhong Zheng, Linear System Theory (Second Edition). Beijing: Tsinghua University Press, 2002, pp. 22-33

[4] G. Blankenstein, R. Ortega. "The Matching Conditions of Controlled Lagrangians and IDA-Passivity Based Control." International Journal of Control, 2002, (75): 645 665

[5] Jiuhe Wang, Peirong Xia, Jinlong Zhang. "Control Strategy of Three-Phase AC/DC Voltage-Source Converters Based on Storage function". 2008 Workshop on Power Electronics and Intelligent Transportation System. pp. 117-121

[6] Jiuhe Wang. "The Nonlinear Control for the Voltage Source PWM Rectifier". Beijing: China Machine Press, 2008, pp.160-163.

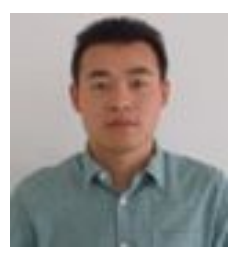

Xiaobin Mu received his M.Sc. degree in electrical engineering from BIST University. His research interests are emerging energy systems.

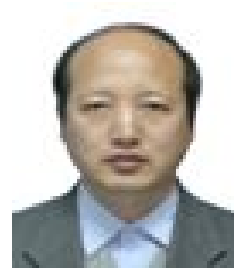

Jiuhe Wang received his $\mathrm{Ph} . \mathrm{D}$ degree in control science and engineering from University of Science and Technology Beijing. His research interests are power electronics and nonlinear control.

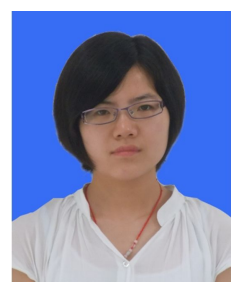

Xueyu Bao received her B.E degree in electronic information engineering from North China Science and Technology University. Her research interests are emerging energy systems and power electronics. 